\title{
Symmetry-breaking charge transfer dynamics of 9,9'-bianthracene revealed by experiment and theory
}

\author{
Changmin Lee ${ }^{1}$, Taecheon $\mathrm{Lyu}^{1}$, Cheol Ho $\mathrm{Choi}^{2}$, and Taiha Joo ${ }^{1, *}$ \\ ${ }^{1}$ Department of Chemistry, Pohang University of Science and Technology (POSTECH), Pohang \\ 37673, Korea \\ ${ }^{2}$ Department of Chemistry, Kyungpook National University, Daegu 41566, Korea
}

\begin{abstract}
Symmetry-breaking charge transfer reaction of 9,9'bianthracene is driven exclusively by the rotational fluctuation of solvents, not including the inertial component in the solvation dynamics. Torsional motion and bond shortening during the reaction were observed.
\end{abstract}

\section{Introduction}

Symmetry-breaking charge transfer (SBCT) has been recognized as an important subject since the observation of SBCT in the photosynthetic reaction centre [1]. SBCT can be a good strategy towards charge separation with small driving force and directional specificity. A representative model system of SBCT is 9,9'-bianthracene (BA). BA has two anthracene units linked directly by a $\mathrm{C}-\mathrm{C}$ single bond. It is reported that the two anthracene rings are orthogonal in the ground state. Upon photoexcitation, the excitation resides in one of the two anthracene rings, which is called locally excited (LE) state, and the LE state undergoes charge transfer (CT) in polar solvents to form positive and negative anthracene rings [2]. The dynamic aspect of the CT reaction is of prime importance, which is the subject of this work. Time-resolved fluorescence (TF) and TF spectra (TFS) in polar solvents were obtained with 50 fs time resolution to attain accurate solvent-dependent reaction rates and nuclear coordinates responsible for the reaction, and compared with the solvation functions. Comprehensive excited-state quantum mechanics (QM)/effective fragment potential (EFP) molecular dynamics (MD) simulation was performed [3]. The simulation matches quantitatively with the experiment and reveals molecular details of the SBCT reaction.

\section{Time-resolved fluorescence spectra of 9,9'-bianthracene}

The absorption and emission spectra of BA in several solvents are shown in Fig. 1(a). Absorption spectra are the same in different solvents. Increased Stokes shifts in solvents with higher polarity indicate the formation of CT state. The TFS of BA in heptane,

\footnotetext{
* Corresponding author: thjoo@postech.ac.kr
} 
acetonitrile, acetone, and ethanol were obtained as a function of time delay. The TFS in the nonpolar solvent heptane is invariant at different times, whereas they show dynamic Stokes shifts in all polar solvents.
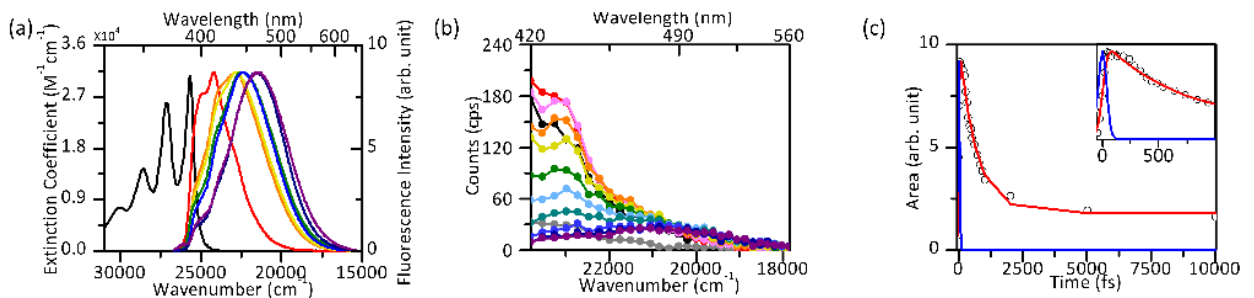

Fig. 1. (a) Absorption (black) and fluorescence spectra of BA in several solvents: red, heptane; orange, propanol; yellow, ethanol; green, methanol; blue, acetone; navy, acetonitrile; purple, ethylene glycol. (b) TFS of BA in acetonitrile: Grey, -50 fs; black, 0 fs; red, 50 fs; pink, 100 fs; orange, $200 \mathrm{fs}$; yellow, 300 fs; green, $500 \mathrm{fs}$; sky-blue, $750 \mathrm{fs}$; cyan, 1 ps; blue, 2 ps; navy, 5 ps; and purple, 10 ps. Excitation wavelength was $380 \mathrm{~nm}$. (c) Area of TFS of BA in acetonitrile: black dots are experiment, red line is the fitting results, and the blue line is the instrumental response function. The inset indicates the same data from -50 fs to $1 \mathrm{ps}$.

Fig. 1b shows the TFS of BA in acetonitrile. The CT band centred around $480 \mathrm{~nm}$ is apparent in the TFS for $t>1 \mathrm{ps}$, although the LE and CT bands cannot be divided because of the dynamic Stokes shifts of both bands. The CT band shows much lower intensity, that is, smaller oscillator strength, than the LE band. Because intensity of the LE fluorescence is about ten times larger, precise CT rate can be readily determined from the decay of the integrated TFS intensity. The TFS area vs. time delay shown Fig. 1c fits well to a single exponential decay of time constant of $640 \mathrm{fs}$, which should be the precise CT time. For all the solvents studied in this work, the obtained CT rate matches quantitatively with the solvation rate [4]. Surprisingly, however, the inertial component present in the solvation function of all liquids does not appear in the dynamics of the SBCT. Therefore, we conclude that the SBCT reaction of BA is coupled with the solvent motion, which should be the full rotational motion of solvents, excluding the inertial component.

\section{Excited-state quantum mechanics/effective fragment potential molecular dynamics simulation}

To attain the molecular details of the SBCT of BA, excited-state QM/EFP MD simulation was implemented. QM part was performed by TDDFT/CAM-B3LYP while tracking the $\mathrm{S}_{1}$ state [5], and EFP-EFP interaction was described by the Lennard-Jones potential and Coulomb interaction [6]. QM-EFP interaction parameter was constructed from the method of localized molecular orbital energy decomposition analysis (LMOEDA) [7]. The QM part of BA and the EFP part of 378 acetonitrile molecules were prepared in a spherical box. After minimizing total energy, ground-state equilibration was done for 100 ps. For the excited-state simulation, a total of 65 points in the time step of 500 fs were selected from 68 to $100 \mathrm{ps}$ as the initial Franck-Condon (FC) points. The CT state was formed within 2 ps in all the MD simulations. The formation of the CT state was verified by the decrease of the oscillator strength and the increase of the dipole moment in the direction of the central C-C bond.

The oscillator strength of the $S_{1}$ state averaged over the 65 trajectories is shown in Fig. 2a black line, which can be compared directly with the TFS area in Fig. 1c. The oscillator strength decreases by the time constant of $1.0 \mathrm{ps}$, in excellent agreement with the experiment. We also calculated the electric field at the centre of the BA molecule in the 
direction of the central $\mathrm{C}-\mathrm{C}$ bond, and the average value over the 65 trajectories is shown in Fig. 2a red line. The rate of change of the electric field is also about $1 \mathrm{ps,} \mathrm{which} \mathrm{is} \mathrm{the} \mathrm{same}$ as the reaction rate. Since internal coordinates of all EFP molecules are fixed, the change of the electric field must be originated from the rotational fluctuation of the solvents. Once the $\mathrm{CT}$ occurs by solvent fluctuation, the CT state further induces solvent reorganization, which in turn further increases the electric field. The simulation fully corroborates the experimental results and the conclusion that the SBCT reaction of BA is coupled entirely with the rotational fluctuation of solvent molecules excluding the inertial component.

(a)

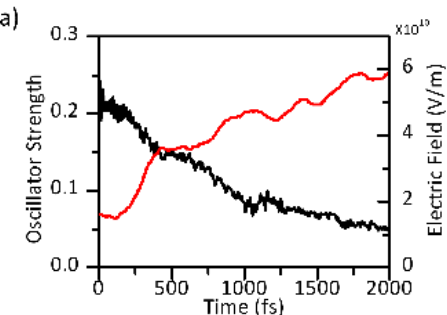

(b)

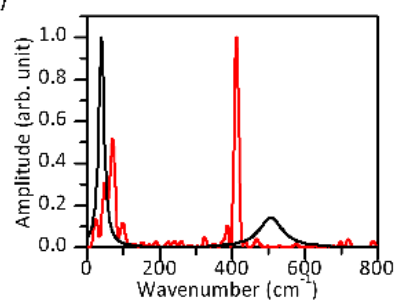

Fig. 2. QM/EFP-MD simulation results. (a) Averaged oscillator strength vs. time (black line) and averaged electric field at the centre of BA in the direction of the central C-C bond. (b) LPSVD result of the TF data of BA in acetonitrile detected at $420 \mathrm{~nm}$ (black line) and the Fourier transform of the residuals of the transition energy between the ground and the $\mathrm{S}_{1}$ state (red line).

The QM/EFP-MD simulation shows that the torsional angle and the length change between the two anthracene rings during the $\mathrm{CT}$ reaction. This implies that the torsional motion and the C-C stretching vibrations can be coherently excited in the LE state. These coherent vibrations can be retrieved by the Fourier transform of the $S_{1}$ transition energy (Fig. 2b, red line) and linear prediction and singular value decomposition of the TF signal of BA in acetonitrile (Fig. 2b, black line) [8].

\section{Conclusion}

Photoinduced SBCT of BA was investigated via TFS and excited-state QM/EFP MD simulation. The CT reaction of BA is coupled entirely with the rotational motion of solvents. However, the inertial component in solvation dynamics does not cause the CT reaction. The torsional motion between two anthracene rings at $40 \mathrm{~cm}^{-1}$ and the stretching motion of the central $\mathrm{C}-\mathrm{C}$ bond at $500 \mathrm{~cm}^{-1}$ were observed in the TF signal, and these motions are also identified in the MD simulation.

\section{References}

1. E. Vauthey, ChemPhysChem 13, 2001 (2012)

2. T. Takaya, H. Hamaguchi, H. Kuroda, K. Iwata, Chem. Phys. Lett. 399, 210 (2004)

3. M.S. Gordon, M.A. Freitag, P. Bandyopadhyay, J.H. Jensen, V. Kairys, W.J. Stevens, J. Chem. Phys. A 105, 293 (2001)

4. M.L. Horng, J.A. Gardecki, A. Papazyan, M. Maroncelli, J. Phys. Chem. 99, 17311 (1995)

5. T. Yanai, D.P. Tew, N.C. Handy, Chem. Phys. Lett. 393, 51 (2004)

6. A.M. Nitkin, A.P. Lyubartsev, J. Comput. Chem. 28, 2020 (2007)

7. P. Su, H. Li, J. Chem. Phys. 131, 014102 (2009)

8. H. Barkhuijisen, R. de Beer, W.M.M. J. Bovée, D. van Ormondt, J. Mag. Reson. 61, 465 (1969) 